\title{
Comparison between the contribution of ellagitannins of new oak barrels and one-year-used barrels
}

\author{
María Navarro $^{1}$, Nikolaos Kountoudakis ${ }^{1}$, Sergio Gómez-Alonso ${ }^{2}$, Esteban García-Romero ${ }^{3}$, Joan Miquel Canals ${ }^{1}$, Isidro \\ Hermosín-Gutíerrez ${ }^{2}$, and Fernando Zamora ${ }^{1}$ \\ ${ }^{1}$ Departament de Bioquímica i Biotecnologia, Facultat d'Enologia de Tarragona, Universitat Rovira i Virgili, C/Marcel.li Domingo, \\ s/n. 43007 Tarragona, Spain \\ ${ }^{2}$ Universidad de Castilla-La Mancha, Instituto Regional de Investigación Científica Aplicada, Campus Universitario s/n, 13071 Ciudad \\ Real, Spain \\ ${ }^{3}$ Instituto de la Vid y el Vino de Castilla-La Mancha, Ctra. Toledo-Albacete s/n, 13700 Tomelloso, Ciudad Real, Spain
}

\begin{abstract}
The influence of the botanical origin (French oak: Quercus petraea and American oak: Quercus $a l b a$ ), toasting level and if the barrel were new of previously used during one year have been studied. Results indicate that French oak released significant higher amounts of ellagitannins than American oak. Toasting level also exert a great influence. The higher the toasting level the lower the ellagitannin concentration in wines. Finally, the use during one year of the barrels drastically decreases the ellagitannin concentration in wines. Consequently, it can be concluded that the origin of oak, the toasting level and especially the previous use of the barrels have a very significant influence on the final ellagitannin concentration in wine, and probably on its sensory impact.
\end{abstract}

\section{Introduction}

High-quality red wines are traditionally aged in oak barrels to improve their sensorial characteristics. Oak aging causes some interesting changes in red wines, leading to color stabilization, lower astringency, and the disappearance of excess vegetative notes [1-4]. The contact with oak wood also enriches the wine in many volatile substances that improve the intensity and complexity of the wine's aroma [5] and also in some non-volatile substances like ellagitannins [6], which contribute to wine texture sensations such as astringency and mouthfeel [7].

The interest of wineries and cooperages in how the botanical origin and the toasting level affect wine's composition and quality has led to numerous studies [8-10]. However, most of them are related with the aromatic aspects and only a few have targeted the study of the ellagitannin release $[11,12]$. Moreover, these latter studies were performed only with new barrels, which entail a lack of information about what happens with used barrels. For that reason, the aim of this study was to compare the contribution of ellagitannins of new oak barrels and one-year-used barrels in order to know the depletion rate of oak wood in these substances.

\section{Materials and methods}

Six American oak (Q. alba) and six French oak $(Q$. petraea) barrels were used in this study. For each type of oak, three toasting levels were used, light, medium and heavy so that there were two barrels for each experimental group. The experience was performed during two years with two red wines of 2012 and 2013 vintages (Cabernet Sauvignon, AOC Tarragona, Spain) which were maintained in the barrels for 12 months. In this way, the first wine was aged in new barrels whereas the second wine was aged in the same barrels which have already been used for a year. After the experience, ellagitannins were analyzed by HPLC [13]. The ellagitannins were identified by matching the retention time and spectral data (DAD$\mathrm{UV}$-vis and MS/MS) with those of authentic standards.

\section{Results and discussion}

Figure1 shows the total and individual ellagitannin concentrations after 12 months of barrel aging in new and 1-year used American and French barrels.

This figure graphically illustrates what really happens after the most common time period for wine aging. These graphics clearly show the importance of the selection of the characteristics of the barrel in terms of their contribution of ellagitannins to wine. Overall, French oak release a significant higher amount of ellagitannins than American oak. The toasting level also exerts a great influence on ellagitannin levels in wine. Specifically the higher the toasting level the lower the ellagitannin content. Finally, the use of the barrel during one year drastically decreases its contribution in ellagitannins to wine during aging in all experimental conditions.

The theoretical sensory impact of the ellagitannins present in the different wines ( $\left.\sum \mathrm{Ei} / \mathrm{Ti}\right)$ was calculated using the individual threshold of the different ellagitannins previously reported [14] in order to show the hypothetic importance of the contribution of the different barrels with regard to this family of phenolic compounds

Figure 2 shows the theoretical contribution of new and used French and American barrels in terms of ellagitannin release. 


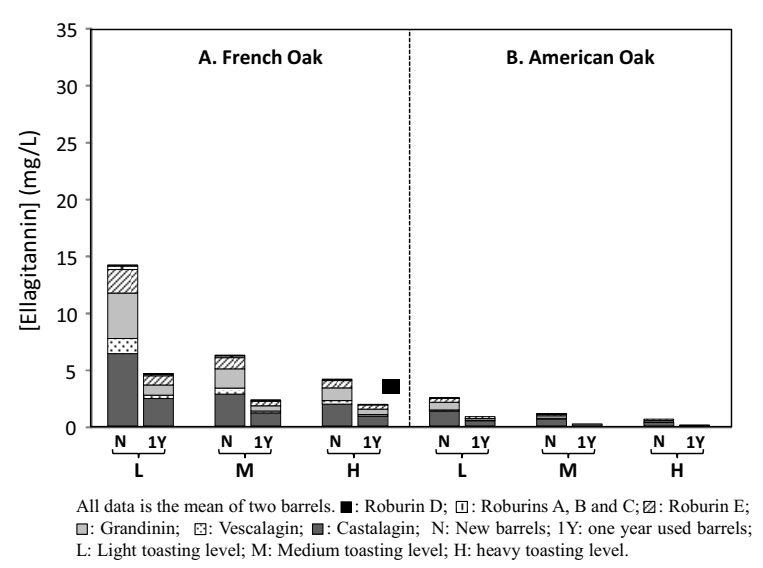

Figure 1. Ellagitannin content of red wine after 12 months of oak aging.

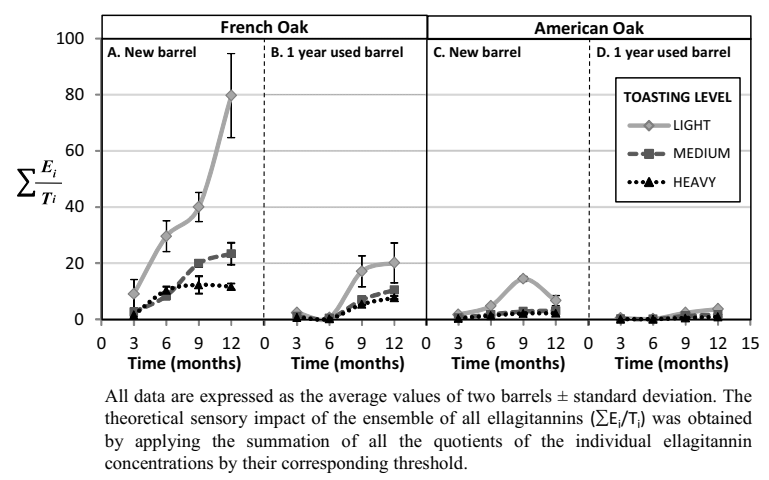

Figure 2. Theoretical sensory impact of ellagitannins in the decolored white wine.

Once again, these results confirms that the sensory contribution of ellagitannins released by French oak are significant higher than in American oak and decrease when the toasting level is higher. Moreover, it seems that the use of the barrels, even by only one year drastically reduce the contribution of ellagitannins during wine aging.

In short, it can be concluded that the origin of oak, the toasting level and especially the previous use of the barrels have a very significant influence on the final ellagitannin concentration in wine, and probably on its sensory impact.

We would like to thank CICYT (Projects AGL2014-56594-C2-1$\mathrm{R}$ and AGL2014-56594-C2-2-R) for its financial support.

\section{References}

[1] Singleton, V. L. (1995). Maturation of wines and spirits: Comparisons, facts, and hypotheses. Am. J. Enol. Vit., 46, 98-115

[2] Garde-Cerdán, T., \& Ancín-Azpilicueta, C. (2006). Review of quality factors on wine ageing in oak barrels. Trends Food Sci. Tech., 17, 438-447

[3] Chira, K., \& Teissedre, P.L. (2013a). Extraction of oak volatiles and ellagitannins compounds and sensory profile of wine aged with French winewoods subjected to different toasting methods: Behaviour during storage. Food Chem., 140, 168-177

[4] Pérez-Juan, P. M., \& Luque de Castro, M. D. (2015). Use of Oak Wood to Enrich Wine with Volatile Compounds. In V.R. Preedy (Ed.), processing and impact on active components in food (pp. 471-481). London: Academic Press

[5] Garde-Cerdán, T., Rodríguez-Mozaz, S., \& AncínAzpilicueta, C. (2002). Volatile composition of aged wine in used barrels of French oak and of American oak. Food Res. Int., 35, 603-610

[6] Chira, K., Zenga, L., Le Floch, A., Péchamat, L., Jourdes, M., Teissedre, P.L. (2015). Compositional and sensory characterization of grape proanthocyanidins and oak wood ellagitannin. Tetrahedron, 71, 2999-3006

[7] Chira, K., Teissedre, P.L. (2013b). Relation between volatile composition, ellagitannin content and sensory perception of oak wood chips representing different toasting processes. Eur. Food Res. Tech., 236, 735-746

[8] Díaz-Maroto, M.C., Guchu, E., Castro-Vazquez, L., de Torres, C., \& Pérez-Coello, M.S. (2008). Aromaactive compounds of American, French, Hungarian and Russian oak woods, studied by GC-MS and GC-O. Flav. Fragr. J., 23, 93-98

[9] Fernández De Simón, B., Martínez, J., Sanz, M., Cadahía, E., Esteruelas, E., \& Muñoz, A.M. (2014). Volatile compounds and sensorial characterisation of red wine aged in cherry, chestnut, false acacia, ash and oak wood barrels. Food Chem., 147, 346-356

[10] Rodríguez-Rodríguez, P., \& Gómez-Plaza, E. (2012). Volatiles in Barrel-Aged Wines. Food Tech. Biotech., 50, 59-65

[11] Jourdes, M., Lefeuvre, D., Quideau, S. (2009). C-Glycosidic ellagitannins and their influence on wine chemistry. In Quideau, S. (Ed) Chemistry and Biology of Ellagitannins. An Underestimated Class of Bioactive Plant Polyphenols (pp 320-365). World Scientific

[12] Navarro, M., Kontoudakis, N., Gómez-Alonso, S., García-Romero, E., Canals, J.M., HermosínGutíerrez, I., Zamora, F. (2016) Influence of the botanical origin and toasting level on the ellagitannin content of wines aged in new and used oak barrels. Food Res. Int., 87, 197-203

[13] Navarro, M., Kontoudakis, N., Giordanengo, T., Gómez-Alonso, S., García-Romero, E., Fort, F., Canals, J.M., Hermosín-Gutíerrez, I., Zamora, F. (2016) Oxygen consumption by oak chips in a model wine solution; Influence of the botanical origin, toast level and ellagitannin content. Food Chem., 199, 822-827

[14] Glabasnia, A., \& Hofmann, T. (2006). SensoryDirected Identification of Taste-Active Ellagitannins in American (Quercus alba L.) and European Oak Wood (Quercus robur L.) and Quantitative Analysis in Bourbon Whiskey and Oak-Matured Red Wines. J. Agric. Food Chem., 54, 3380-3390 